\title{
Heike Kahlert
}

\section{Demographische Frage, „Qualität” der Bevölkerung und pronatalistische Politik - ungleichheitssoziologisch betrachtet ${ }^{1}$}

\section{Europa vor der „demographischen Herausforderung”}

Europa droht in seiner Bevölkerung zu schrumpfen: Schenkt man etwa dem Basisszenario der Bevölkerungsschätzungen von Eurostat vom Dezember 2004 Glauben, so wird EU-Europa bis 2025 nur noch leicht anwachsen, um danach eine zunehmend negative Bevölkerungsentwicklung aufzuweisen. ${ }^{2}$ Dem „Grünbuch“ der Kommission der Europäischen Gemeinschaften zum demographischen Wandel zufolge verzeichneten 55 der 211 Regionen der (damals) 15 Mitgliedsstaaten der Europäischen Union schon in der zweiten Hälfte der 1990er Jahre einen Bevölkerungsrückgang; dies gelte auch für die meisten Regionen der neuen Mitgliedstaaten (35 von 55), bedingt durch natürlichen Rückgang und Nettoabwanderung. Die Europäische Kommission zeigt sich von der angenommenen Bevölkerungsentwicklung alarmiert, denn der Rückgang der Bevölkerung im erwerbsfähigen Alter (15-64 Jahre) dürfte ihren Prognosen zufolge zwischen 2005 und 2030 bei 20,8 Millionen Personen liegen (Kommission 2005: 2).

Der mit dem Altern der europäischen Bevölkerung verbundene demographische Wandel könnte der Kommission zufolge „das jährliche 'potenzielle Wachstum' des BIP in Europa von heute [2005] 2-2,25\% auf 1,25\% im Jahre 2040 drücken. Unternehmergeist und Initiativfreudigkeit unserer Gesellschaft wären dadurch ebenfalls beeinträchtigt“ (Kommission 2005: 3). Unmissverständlich wird hier deutlich, dass Alterung und Schrumpfung der Bevölkerung in den westlichen Gesellschaften erheblichen Anlass zur ökonomisch begründeten Besorgnis geben, denn ihr Fortschrittsverständnis ist untrennbar mit dem Prinzip des Wachstums verbunden: „Noch nie gab es Wirtschaftswachstum ohne Bevölkerungswachstum“ (ebd.: 5). Dieser Zusammenhang wird im „Grünbuch“ in der ökonomisch-neoliberal geprägten Sprache der Europäi-

1 Für konstruktive Anmerkungen zu einer früheren Fassung dieses Beitrags danke ich Peter A. Berger.

2 Das Szenario nennt folgende Zahlen: 458 Millionen Einwohner im Jahr 2005, 469,5 Millionen Einwohner im Jahr 2025 (+2\%) und 468,7 Millionen Einwohner im Jahr 2030. 
schen Kommission als Angst vor dem Verlust der erreichten Stellung und Bedeutung Europas in der Weltwirtschaft ausgedrückt, aber auch als Sorge um die Finanzierbarkeit der Sozialsysteme, insbesondere der Alterssicherung.

Als Antwort auf die „demographische Herausforderung“ will die Europäische Kommission „drei wesentliche Prioritäten verfolgen: Wieder auf den Weg des demografischen Wachstums kommen (...), Sicherstellung eines Gleichgewichts zwischen den Generationen, (...) Schaffung neuer Übergänge zwischen den Lebensabschnitten“ (ebd.: 3, 12). Schwerpunkte der politischen Maßnahmen zur Sicherung der „finanziellen und sozialen Nachhaltigkeit“ sollen in der entschlossenen Umsetzung der Agenda von Lissabon (Modernisierung der Sozialsysteme, Steigerung der Beschäftigungsquote insbesondere von Frauen, Jugendlichen und älteren Arbeitnehmern, Investitionen in das so genannte $\mathrm{Hu}-$ mankapital, verstärkte Forschung, Innovation und Produktivitätssteigerung), in „innovative[n] Maßnahmen zur Förderung der Geburtenrate“ sowie einem „kontrollierten Rückgriff auf die Zuwanderung“ (ebd.: 3, 12) liegen.

Aus demographischer Sicht besteht weitgehende Einigkeit darüber, dass kurzund mittelfristig in Europa - wie auch in anderen westlichen Gesellschaften bestenfalls die Stabilisierung der Bevölkerungszahlen zu erreichen, eher aber mit einem Schrumpfen der Bevölkerung zu rechnen ist. Infolgedessen ist die Frage nach der „Qualität“ der Bevölkerung, verstanden im Sinne der aus EUSicht zu leistenden Investitionen in das Humankapital, auf die transnationale politische Agenda, aber auch auf die der europäischen Nationalstaaten gerückt.

In Reaktion auf die zu erwartenden demographischen Veränderungen messen die meisten europäischen Staaten der Familie (und der Familienpolitik) eine höhere Priorität bei als je zuvor und nehmen entsprechende Umgestaltungen in ihren politischen Programmen vor (Daly 2004: 136). Die Lösung der demographischen Frage ist zu einem politischen Querschnittsthema geworden und umfasst dabei - neben der Familienpolitik - so verschiedene Politikfelder wie Jugend-, Senioren-, Bildungs-, Arbeitsmarkt-, Forschungs- und Zuwanderungspolitik. Am Beispiel des in Deutschland neuerdings in Reaktion auf die „demographische Herausforderung“ entwickelten Konzepts einer „nachhaltigen Familienpolitik“ argumentiere ich im Folgenden unter Rückgriff auf einschlägige politische Dokumente und Verlautbarungen, dass die demographische Frage, die soziale Frage und die Geschlechterfrage eng miteinander verschränkt sind. In der „nachhaltigen Familienpolitik“ wird, so meine These, die Chance zur Modernisierung des konservativen deutschen Wohlfahrtsstaats (vgl. Esping-Andersen 1996) vertan.

Im Fokus der Analyse der diesem Ansatz zugrunde liegenden Orientierungen stehen die Kategorien Klasse (bzw. Schicht) und Geschlecht. Dieses Politikkonzept ist, wie ich erstens zeigen werde, stark pronatalistisch ausgerichtet. Pronatalismus verstehe ich in Anlehnung an Anne Hélène Gauthier (1996: 
203) als aktive Geburtenförderung, die Programme des Mutterschafts"urlaubs“, die Förderung von Kinderbetreuungseinrichtungen und finanzielle Unterstützungen für Familien in den Vordergrund des familienpolitischen Handelns stellt. Damit sollen Fertilitätshindernisse, insbesondere in Gestalt der strukturellen Unvereinbarkeit von Beruf und Familie, reduziert werden. Zweitens werde ich darlegen, dass sich die pronatalistische Orientierung der „nachhaltigen Familienpolitik“ explizit mit dem Ziel der Sicherung bzw. Steigerung der „Qualität“ der Bevölkerung verknüpft. Dem Konzept der „nachhaltigen Familienpolitik“ zufolge soll die Geburtenförderung in Deutschland sozial differenziert erfolgen. Adressatinnen von herausragendem Interesse sind für sie die hoch qualifizierten Frauen, sprich: die Akademikerinnen, die neuerdings als besonders zu fördernde erwerbstätige Mütter in den politischen Aufmerksamkeitshorizont geraten sind. Die damit scheinbar sukzessiv näher rückende Verwirklichung der Frauenemanzipation bzw. eines egalitären Geschlechterverhältnisses zumindest für die besser Verdienenden ist jedoch, so mein dritter Argumentationsschritt, lediglich eine Nebenfolge der kindzentrierten familienpolitischen Anstrengungen. Zwar pflegt die „nachhaltige Familienpolitik“ auch eine gleichstellungsorientierte Rhetorik, misst dem Gleichstellungsziel faktisch jedoch allenfalls nachrangige Bedeutung zu. Im abschließenden Ausblick wird die pronatalistische Familienpolitik Deutschlands aus ungleichheitssoziologischer Sicht schließlich in international vergleichender Perspektive problematisiert.

\section{2. „Nachhaltige Familienpolitik” - zur Karriere eines neuen Politikkonzepts}

Die Familienpolitik, seit ihrer Etablierung als eigenständiges Politikfeld eher ein Stiefkind der deutschen Politik, hat seit dem Wahlkampf 2002 „einen zentralen Stellenwert“ (BMFSFJ 2005) im Regierungshandeln bekommen unabhängig von der Couleur der Regierenden. Dieser Bedeutungszuwachs geht mit einer Neuausrichtung und stärkeren Orientierung der Familienpolitik „an bevölkerungspolitischen Erfordernissen“ (BMFSFJ u.a. 2004: 19) einher: „Familienpolitik wurde in Deutschland (...) bisher überwiegend als finanzielle Unterstützung von Familien verstanden - die Entwicklung der Bevölkerung spielte weniger eine Rolle. Die Gesetze zur Familienförderung wurden von verschiedenen Ressorts erlassen. Ein konsistenter Politikansatz fehlt bislang ebenso wie eine umfassende Auswertung der einzelnen Maßnahmen. (...) Inzwischen setzt sich das Bewusstsein immer mehr durch, dass eine 'bevölkerungsorientierte Familienpolitik' die Geburtenrate wieder steigern könnte. Ziel dieser Überlegungen ist es, Familien nicht mehr ausschließlich materiell zu fördern, sondern die Bedingungen für das Kinderkriegen allgemein zu verbessern.“ (BMFSFJ u.a. 2004: 8) Die Leitlinie einer derart „nachhaltigen Politik 
für Familien“ lautet: „mehr Kinder in den Familien und mehr Familie in der Gesellschaft“ (Bundesregierung 2006: 7).

Angesichts der nationalsozialistischen Bevölkerungspolitik stellt die in diesen neueren programmatischen Dokumenten aufscheinende pronatalistische Ausrichtung in der Familienpolitik aus westdeutscher Sicht durchaus einen Tabubruch dar; aus ostdeutscher Sicht hingegen ist sie vertraut, denn auch die DDR betrieb eine aktiv geburtenfördernde Politik. Zentrale Anstöße zur „demographische[n] Wende“ (Oestreich 2005: 11) bzw. zum Aufstieg des Pronatalismus in der gesamtdeutschen Familienpolitik gaben Endberichte politischer Kommissionen, vor allem der Enquête-Kommission „Demographischer Wandel“ (Deutscher Bundestag 2002) und der „Kommission für die Nachhaltigkeit in der Finanzierung der sozialen Sicherungssysteme" (BMGS 2003), letztere besser bekannt als Rürup-Kommission³. Sie erhöhten die öffentliche Aufmerksamkeit für die sozialpolitischen und sozialen Folgen der ökonomischen Krise und der gesellschaftlichen Alterung.

Damit war in der Öffentlichkeit ein nachhaltiger demographischer Alarmismus in Gang gesetzt. Sollten die Berichte der Regierungskommissionen dafür sensibilisieren, dass der deutsche Sozialstaat aufgrund der alternden Bevölkerung an seine Leistungsgrenzen stoße und der Generationenvertrag nicht mehr funktioniere, so suggerierte die zeitgleich vorgelegte „PISA 2000“-Studie zudem, dass die „Qualität“ des Humankapitals der ohnehin knapper werdenden Heranwachsenden allenfalls als mittelmäßig einzuschätzen sei: In internationalen Vergleichsuntersuchungen der OECD zum Leistungsstand von Schülerinnen und Schülern hatten die deutschen Jugendlichen im Hinblick auf die getesteten Kompetenzen vergleichsweise schlecht abgeschnitten und etwa in der Lesekompetenz nur den 21. Platz (von 32) belegt (vgl. Deutsches PISAKonsortium 2001). Der „PISA-Schock“ (Oestreich 2005: 11) und die sich abzeichnende Überalterung der Gesellschaft machten darauf aufmerksam, dass die erreichte wirtschaftliche Position Deutschlands im Weltgefüge wie auch das erreichte Niveau der sozialstaatlichen Sicherung in Gefahr seien (vgl. Kaufmann 2005).

Auf die so gestellte „demographische Frage“ antwortet die Bundesregierung mit der Erkenntnis: „Was wir brauchen, ist eine nachhaltige Bevölkerungsentwicklung für unser wirtschaftliches Wachstum und für die nachhaltige Stabilisierung der Grundlagen sozialer Sicherheit." (Bundesregierung 2006: 21, Hervorh. i. Orig.) Sie wird dabei durch sozialwissenschaftliche Politikberatung angeregt und unterstützt - vor allem in Gestalt des Volkswirtschaftlers und

3 Diese unterbreitete Vorschläge, „wie wir die Sozialsysteme zukunftsfest machen können. Und zukunftsfest heißt: sicher und bezahlbar“ - angesichts der „schwierige[n] konjunkturelle[n] Lage und [der] Tatsache, dass immer weniger junge Menschen das soziale Netz für immer mehr ältere Menschen halten" (http://www.bmas.bund.de/BMAS/Navigation/SozialeSicherung/berichte,did=105578.html). 
„Wirtschaftsweisen“ Bert Rürup sowie des (Familien-)Soziologen Hans Bertram, die beide nicht nur den Vorsitz zentraler Kommissionen der Bundesregierung innehatten, sondern für diese ebenso Weg weisende Expertisen zur Familienpolitik erstellten.

\section{Konturen der „nachhaltigen Familienpolitik”}

Laut Rürup/Gruescu verfolgt die „nachhaltige Familienpolitik“ zwei konkrete Ziele: erstens „eine ausreichende Kinderzahl, die der Alterung der Gesellschaft entgegenwirkt“ und „durch eine Erhöhung der Geburtenrate zu einer langfristig stabilen Bevölkerung führen kann“ (Hervorh. i. Orig.), und zweitens „eine Erhöhung der Frauenerwerbstätigenquote, um das Erwerbspersonen- und Fachkräftepotenzial (...) auch kurzfristig“ zu erhöhen bzw. zu stabilisieren (Rürup/ Gruescu 2003: 7, 56). Diese Ziele korrespondieren mit den eingangs erwähnten politischen Maßnahmen der Europäischen Kommission insofern, als sie die Agenda von Lissabon und die Geburtenförderung aufgreifen. Politisch gesteuerte Zuwanderung, wie von der Europäischen Kommission ebenfalls vorgeschlagen, gehört hingegen (bisher) nicht zu diesem Politikkonzept, obwohl diese Maßnahme am Rande ebenfalls von Rürup/Gruescu (2003: 55f) benannt wird.

Den sowohl kurz- wie langfristigen Zielsetzungen entsprechend plädieren Rürup/Gruescu (2003: 76) für einen „Policy-Mix“, der eine Kombination aus einem einkommensabhängigen Elterngeld sowie einem flächendeckenden, flexiblen und qualitativ hochwertigen Angebot an Kinderbetreuungsmöglichkeiten - auch für Kinder unter drei Jahren - und einer Flexibilisierung starrer Arbeitszeitmuster vorsieht und von Bertram und Mitarbeiterinnen näher als „Dreiklang von Zeitpolitik, finanzieller Transferpolitik und Infrastrukturpolitik“ (Bertram u.a. 2005: 1) ausgearbeitet wird. Der von Rürup/Gruescu entworfene Maßnahmenkatalog spiegelt detailgenau die eingangs als pronatalistisch charakterisierte Politikorientierung in Gestalt von finanzieller Unterstützung von Familien, Kinderbetreuungseinrichtungen und anderen Maßnahmen zur Verbesserung der Vereinbarkeit von Beruf und Familie wider.

Hinzu kommt die Weiterung der zeitpolitischen Dimension um eine politisch vorzunehmende Umgestaltung der Lebensläufe von Frauen und Männern, die von der Sachverständigenkommission Siebter Familienbericht - unter dem Vorsitz von Hans Bertram - entwickelt wurde (vgl. Sachverständigenkommission 2005). Bedeutsame Elemente des Lebenslaufs seien das Ergebnis politischer Entscheidungen, die beispielsweise auch Einfluss auf die Familiengründung hätten (vgl. Bertram u.a. 2005: 45). So trügen etwa die staatlich geregelte Dauer der schulischen und beruflichen Ausbildung sowie die damit verbundene lange materielle Abhängigkeit junger Frauen und Männer von ihren Eltern dazu bei, dass insbesondere bei Hochqualifizierten das Zeitfenster für eine Familiengründung zwischen dem Erlangen ökonomischer Unabhängigkeit 
und dem Ende der fertilen Phase ausgesprochen knapp und daher die Kinderlosigkeit besonders hoch sei.

Die skizzierten Maßnahmenkataloge verdeutlichen, was „Nachhaltigkeit“ im Zusammenhang mit Familienpolitik meinen soll: Die Zukunft der Gesellschaft hängt demnach im Wesentlichen von der Erhöhung der Geburtenrate und der Ausschöpfung der (knapper werdenden) Humanressourcen für die ökonomische Entwicklung der Gesellschaft ab. Darüber hinaus geht es darum, „dass die nachwachsende Generation die Verpflichtungen, die ihr die gegenwärtige Generation hinterlässt, auch als Verpflichtung annimmt und es akzeptiert, dafür zu arbeiten und Leistungen zu erbringen“ (Bertram u.a. 2005: 7f.) - etwa in Form persönlicher Fürsorge und Unterstützung für die ältere Generation. Die Zukunftssicherung durch „nachhaltige Familienpolitik“ zielt darauf, „junge Erwachsene, Eltern und Kinder so zu unterstützen, dass sie das eigene $\mathrm{Hu}-$ mankapital entwickeln können, gleichzeitig aber in die eigene individuelle $\mathrm{Zu}$ kunft und in die Zukunft der Partnerschaft sowie in die Zukunft der eigenen Kinder investieren können“ (ebd.: 9). Die Legitimation für diese Politik wird nicht primär darin gesehen, „Geburtenraten in die eine oder andere Richtung zu beeinflussen. Vielmehr ist der zentrale Sinn solcher Maßnahmen (...), dass Staat und Gesellschaft hier zumindest teilweise sicherstellen können, dass auch zukünftige Generationen einen Gestaltungsspielraum für ihre eigene persönliche Zukunft haben“ (ebd.: 48).

Das Nachhaltigkeitsverständnis der ehemaligen Familienministerin Renate Schmidt verknüpfte die referierte ökonomische, familiensoziologische und demographische Expertise in geradezu idealtypischer Weise: „Nachhaltigkeit bedeutet für Familien verlässliche Optionen zur Realisierung von Lebensplänen, die heute bei beiden Geschlechtern in hohem Maße Balancen von Erwerbsarbeit und Familie beinhalten. Nachhaltigkeit ermöglicht generationsübergreifende Lebenspläne in den Familien bei gleichzeitiger Sicherung der Ressourcen Bindung und Fürsorge. Für die Gesellschaft ermöglicht das Leitbild Nachhaltigkeit eine dauerhafte Entwicklung. Erziehung und Bildung bewahren und entwickeln unser Humanvermögen, das wiederum ökonomisches Wachstum, soziale Sicherung und Wohlstand gewährleistet. Dauerhaftigkeit bezieht sich auch auf demographische Bestandssicherung. Die Kinderwünsche liegen bei 1,8 - realisiert werden 1,3 -, hilfreich wären 1,7. Dieses Ziel ist mittelfristig erreichbar, wird in vergleichbaren Ländern auch erreicht." (Schmidt 2005: 3) Die mittelfristige Zielgröße für die „nachhaltige Familienpolitik“ liegt somit bei einer Geburtenrate von 1,7 Kindern pro Frau - also unter der gesellschaftlichen Bestandssicherung $(2,1)$, aber über der in Deutschland derzeit zu verzeichnenden Geburtenrate $(1,3)$. Im Hinblick auf die Quantität der Bevölkerung geht die Politik mithin davon aus, den gesellschaftlichen Schrumpfungsprozess zwar verlangsamen, aber nicht gänzlich aufhalten oder gar umkehren zu können. 
Einigkeit besteht in diesem Zusammenhang darüber, dass die Familienpolitik die Bevölkerungsentwicklung beeinflussen kann. Regierungsberater Rürup schlussfolgert im Kontext einer Bestandsaufnahme der Familienpolitiken im internationalen Vergleich, dass die politische Steuerungsfähigkeit des Reproduktionsverhaltens von Menschen außer Frage stehe (Rürup/Gruescu 2003: 47). Das Bundesfamilienministerium in Allianz mit dem Institut der Deutschen Wirtschaft (IW) und dem Bundesverband der Deutschen Industrie (BDI) artikuliert den Steuerungsoptimismus etwas vorsichtiger: „Eindeutige Effekte der Familienpolitik auf die Geburtenrate konnten bisher nicht empirisch nachgewiesen werden. Die Menschen bekommen Kinder nicht allein wegen des Kindergeldes oder weil Kinderbetreuung angeboten wird. Dennoch bestehen Plausibilitäten, wie ein Blick in andere Länder zeigt. (...) Die Art der Ausgestaltung der Elternzeit, die Kinderbetreuungsinfrastruktur und die Besteuerung (Haushalts- versus Individualbesteuerung) sind wichtige Ansatzpunkte, wie ein Staat das Verhalten der Familien beeinflusst." (BMFSFJ u.a. 2004: 17, 19). Staatliche Intervention sucht jedoch nicht nur die Quantität der Bevölkerung zu steuern, sondern reguliert über die öffentliche Bildung und Erziehung auch die "Qualität“ des Humankapitals. Im nächsten Schritt soll daher der sozialstrukturellen Dimension der pronatalistischen Ausrichtung der neuen deutschen Familienpolitik nachgegangen werden.

\section{Pronatalismus im Zeichen der "zweiten deutschen Bildungskatastrophe"}

In schrumpfenden Gesellschaften ist aus ökonomischer Perspektive die „Qualität“ der Bevölkerung von wachsender Bedeutung: „Geht man davon aus, dass das Humankapital für den technischen Fortschritt von enormer Bedeutung ist, ergibt sich aufgrund der Bevölkerungsschrumpfung ein negativer Effekt für die Quantität des Humankapitals. Somit kommt der Qualität des Humankapitals eine Schlüsselfunktion zu.“ (Rürup/Gruescu 2003: 54). Dieselben Autoren thematisieren die „Qualitätsfrage“ im Hinblick auf alternde Belegschaften: Da ältere Arbeitnehmer seltener an Weiterbildungsmaßnahmen teilnähmen als jüngere, könne man von einer teilweise sinkenden Qualität des Humankapitals im Alter ausgehen; zudem nehme mit zunehmendem Lebensalter die Risikoaversion zu und Innovationstätigkeiten erlahmten (ebd.: 55). Im Blickpunkt des Bundesfamilienministeriums steht hingegen die Bildung und Erziehung der - zahlenmäßig kleineren - nachwachsenden Kohorten: „In quantitativer wie auch in qualitativer Hinsicht führt der Bevölkerungsrückgang auch zu einem Rückgang des insgesamt verfügbaren Potenzials an Wissen und Fähigkeiten. Deshalb muss sehr frühzeitig in die Qualifikationen des zahlenmäßig knapper werdenden Nachwuchses investiert werden." (BMFSFJ u.a. 2004: 16). Die Bildungsprozesse der nachwachsenden Generation seien für die Sicherung 
der Zukunft des Humanvermögens von zentraler Bedeutung.

Auf das im internationalen Schulleistungsvergleich als mittelmäßig identifizierte Humankapital des „knapper werdenden Nachwuchses“ hatten eindrücklich die auch öffentlich breit diskutierten Ergebnisse der PISA-Studie zu Basiskompetenzen von Schülerinnen und Schülern aufmerksam gemacht (vgl. Deutsches PISA-Konsortium 2001). Als besonders bemerkenswert und für die Familienpolitik besonders relevant an diesen Ergebnissen hatte sich herausgestellt, dass in keinem anderen untersuchten Land der systematische Zusammenhang zwischen familiären Lebensverhältnissen (Berufs- und Bildungsstatus sowie, getrennt ausgewiesen, Migrationshintergrund der Eltern) so eng sei wie in Deutschland, mit der Folge, dass 22,5 Prozent der untersuchten Jugendlichen zur potenziellen Risikogruppe der schwachen Leserinnen und Leser gehörten. Angesichts dieser bildungspolitisch alarmierenden Ergebnisse wird nunmehr eine „zweite deutsche Bildungskatastrophe“ (z.B. Wissenschaftlicher Beirat 2002: 20) ausgerufen, als deren Hauptprotagonist der „muslimische Migrantensohn aus der Großstadt“ gilt. Nicht erst seit „PISA“ ist bekannt, dass die Bildungsexpansion aus ungleichheitssoziologischer Sicht nur bedingt erfolgreich war: Vor allem herkunftsbedingte Bildungsungleichheiten konnten nur unzureichend abgebaut werden, hinsichtlich der geschlechtlichen Bildungsungleichheit werden zusehends die Jungen zu Benachteiligten, und durch globalisierungsbedingte Migrationsprozesse wird die ethnische Bildungsungleichheit zunehmend offensichtlicher. Dauerhafte Bildungsungleichheiten scheinen zur „sozialen Frage des 21. Jahrhunderts“ oder doch zumindest zu einem der wichtigsten Probleme der Zukunft geworden zu sein (Becker/Lauterbach 2004: 9; Geißler 2005).

Die bildungspolitischen Diskussionen in Reaktion auf die Ergebnisse der PISA-Studien nahmen und nehmen vor allem institutionelle Kontexte wie Kinderkrippen und -gärten, die vorschulische Betreuung und das Schulsystem in den Blick. Der Wissenschaftliche Beirat beim Bundesfamilienministerium hebt jedoch hervor, dass Bildung nicht nur in den institutionellen Kontexten des Bildungssystems, sondern auch in den informellen Kontexten der Herkunftsfamilie und des Beziehungsgeflechts der Gleichaltrigen erfolge. Dabei komme der Familie eine „gewichtige Rolle“ im Bildungsprozess zu (Wissenschaftlicher Beirat 2002: 41). Die Familie sei „eine Bildungsinstitution eigener Art“, der „ursprüngliche und begleitende Ort der Bildung von Humanvermögen“ (ebd.: 27, 9), und wirke sich auch auf die Wahl der Schulform und den Schulerfolg der Kinder und Jugendlichen aus. Zu Recht wird in diesem Zusammenhang betont, dass Eltern in vieler Hinsicht die ersten Lehrerinnen und Lehrer ihrer Kinder seien: Sie fördern diese vorschulisch und bereiten sie auf die Schule vor, die wiederum auf die aktive Mithilfe der Eltern angewiesen ist, wenn sie erfolgreich arbeiten will. Dabei besteht ein enger Zusammenhang zwischen der sozialen Schicht der Eltern und den erworbenen Kompetenzen 
der Schülerinnen und Schüler (Baumert/Schümer 2001: 372), die elterlichen Bildungsaspirationen spielen eine gewichtige Rolle für den Bildungserfolg der Kinder. Bildungs- und Familienpolitik, so das Fazit des Beirats, müssten folglich eng aneinander gekoppelt werden.

\section{Die demographische Frage als soziale Frage: Bildungs- ungleichheiten und sozial differenzierender Pronatalismus}

Weder in den PISA-Studien noch im hier zitierten Gutachten des Wissenschaftlichen Beirats für Familienfragen (der im Übrigen in Verklärung der familialen Realitäten hinsichtlich der geschlechtlichen, zumeist ungleichen Arbeitsteilung nur von „Eltern“ redet, statt zwischen Müttern und Vätern zu unterscheiden) geht es explizit um die demographische Frage. Doch lassen sich wie die jüngere Entwicklung zeigt - die entsprechenden Befunde und Argumente auch bevölkerungspolitisch verwenden. Unter Berufung auf beide Quellen wendet die Bundesregierung die Forderung nach einer Kopplung von Bildungs- und Familienpolitik pronatalistisch und sozial differenzierend, indem sie insbesondere die besser gebildeten Frauen in den Blick nimmt: „Die PISAStudie hat für Deutschland einen im Vergleich zu anderen Ländern ausgesprochen großen Abstand der Kompetenzen von Kindern mit Müttern mit geringer Bildung zu Kindern ausgemacht, deren Mütter sehr hoch qualifiziert sind. Dies ist umso bedenklicher, als sich ein hoher Bildungsstandard der Mutter auf die Kompetenz der Kinder positiv auswirkt und damit die Studierfähigkeit junger Menschen befördert. (...) Eine anhaltend hohe Kinderlosigkeit unter Akademikerinnen kann die bildungspolitischen Probleme weiter verschärfen und zu Engpässen beim Fach- und Führungskräfte-Nachwuchs führen.“ (BMFSFJ u.a. 2004: 16). Das Argument könnte auch so ausgedrückt werden: In Deutschland besonders erwünscht sind Kinder von hoch qualifizierten Müttern, denn der im Zusammenwirken von Familie und höherem Bildungsstandard gebildete Nachwuchs ist angesichts knapper werdender Humanressourcen von besonderem Interesse für die Zukunft des Wirtschaftsstandorts Deutschland.

Zwar wird derzeit nicht zuletzt aufgrund solcher Überlegungen in Deutschland durchaus auch in die Verbesserung des öffentlichen Bildungswesens investiert, z.B. in den Ausbau einer qualifizierten Ganztagsbetreuung für Kinder im Alter von eins bis sechs Jahren. Noch bemerkenswerter ist aber, dass mit dem ebenfalls zum Maßnahmenkatalog der "nachhaltigen Familienpolitik“ gehörenden, zum 1.1.2007 von der Großen Koalition eingeführten einkommensabhängigen Elterngeld nun „eine weitere heilige Kuh deutscher Familienpolitik“ geschlachtet worden ist - der Grundsatz nämlich, „dass jedes Kind dem Staat gleich viel wert sein müsse“ (Siems 2004). Dieser Tabubruch wird offen kommuniziert: So warnte Familienministerin Schmidt etwa in einem In- 
terview mit dem DeutschlandRadio Berlin vor der „demographischen Katastrophe“ und der „Bildungskatastrophe für unser Land, wenn in den bildungsnahen Schichten immer weniger oder keine Kinder geboren werden" (Schmidt/ Sagenschneider 2004). Das einkommensabhängige Elterngeld zielt dementsprechend explizit auf die besser Verdienenden: Es soll die Gebär- und Zeugungsfreudigkeit hoch qualifizierter Frauen und Männer fördern, die in Deutschland bislang vergleichsweise häufig kinderlos bleiben. Und während das Elterngeld an der Kompensation der Opportunitätskosten von Kindern vor allem für die besser gestellten Doppelverdiener ansetzt, wird in zeitpolitischer Hinsicht zugleich darüber nachgedacht, wie hoch qualifizierte Frauen zur „frühen Mutterschaft“ (Allmendinger/Dressel 2005: 27) gebracht werden können, also dazu, bereits vor dem oder im Studium ein Kind oder mehrere Kinder zu gebären. Damit würde nicht nur die Generationenfolge verkürzt, sondern auch die Chance auf Mehrfachmutterschaften von Akademikerinnen erhöht - bisher gehören die hoch qualifizierten Frauen, wenn sie überhaupt Mütter werden, eher zu den „Spätgebärenden“, die ihr erstes (und dann nicht selten einziges) Kind ab dem 35. Lebensjahr bekommen.

Es gibt viele gute Gründe dafür, dass die vor allem in Westdeutschland noch immer problematische Vereinbarkeit von Beruf und Familie ${ }^{4}$ verbessert und das in vielerlei Hinsicht als historisch überholt erscheinende deutsche Bildungssystem reformiert wird, z.B. hinsichtlich seiner Dreigliedrigkeit mit der Anforderung an biographisch (zu) frühe Bildungswegentscheidungen oder auch hinsichtlich der erwarteten aktiven Mithilfe der Eltern bzw. Mütter bei eigentlich schulischen Aufgaben; entsprechende Politikansätze sind überfällig und sollen hier keineswegs generell kritisiert werden. Auch ist es wichtig, auf die „bildungspolitische Bedeutung der Familie“ (Wissenschaftlicher Beirat 2002) aufmerksam zu machen und eine stärkere Verzahnung von Elternhaus und Bildungsinstitutionen zu fordern. Aus ungleichheitssoziologischer Sicht liegt ein wesentliches Politikum der „nachhaltigen Familienpolitik“ jedoch in der darin aufscheinenden Verknüpfung zwischen der angestrebten Geburtenförderung und der expliziten sozialen Differenzierung, die die soziale Ungleichheit als vermeintlich „natürliche“ und folglich politisch nicht zu beeinflussende Tatsache akzeptiert. Der Abbau bestehender sozialer Ungleichheiten, immerhin ja ein (einstmaliges?) Grundprinzip des Wohlfahrtsstaats, scheint also angesichts der durch den demographischen Wandel antizipierten ökonomischen und fiskalischen Bedrohungen nicht (mehr) auf der politischen Agenda zu stehen. Der pronatalistische Ansatz der „nachhaltigen Familienpolitik“ hat aber auch geschlechterpolitische Implikationen, die nun etwas näher beleuchtet werden.

4 Dabei bezieht sich die innerdeutsche Differenzierung vor allem auf die in Ostdeutschland aufgrund der anderen politischen Tradition faktisch noch immer häufiger vorhandenen Kinderbetreuungseinrichtungen. 


\section{Der Abschied vom Ernährermodell als Nebenfolge pronatalistischer Politik}

Bertram u.a. (2005: 7) fordern in ihrem Gutachten im Auftrag des Bundesfamilienministeriums, die „nachhaltige Familienpolitik“ müsse so angelegt sein, dass sie unterschiedliche Lebensvorstellungen und individuelle Planungen zur Familiengründung und -erweiterung unterstütze. Dabei weisen Bertram u.a. unter Rückgriff auf jugend- und geschlechtersoziologische Forschungsergebnisse darauf hin, dass sich die Motive und Einstellungen junger Frauen in Bezug auf Kinder und Beruf deutlich von den männlichen Lebensentwürfen unterschieden. Zudem stellten die jungen Frauen keine in sich homogene Gruppe dar, sondern hätten eine Vielfalt an Lebensplänen und konkurrierenden Interessen, in denen Familie und Beruf höchst unterschiedliche Bedeutung zukäme. Im Anschluss an die Präferenztheorie Catherine Hakims (2000) differenzieren sie zwischen den Lebensstilen der ausschließlichen Berufsorientierung, der Familien- und Haushaltsorientierung sowie der adaptiven Orientierung (Vereinbarkeitsmodell). Eine Familienpolitik, die diese unterschiedlichen $\mathrm{Zu}$ kunftsvorstellungen nicht reflektiert und von einer homogenen Motivlage bei allen Frauen ausgeht, würde demzufolge „kaum Erfolg haben können“ (Bertram u.a. 2005: 16).

Im Kern interessiert sich die „nachhaltige Familienpolitik“ jedoch vor allem für die hoch qualifizierten Frauen als Arbeitskräfte und (potenzielle) Mütter: Akademikerinnen ohne Kinder werden derzeit aufgrund ihrer Kinderlosigkeit stigmatisiert, während Mütter mit niedrigem Bildungsstatus als Problem dargestellt werden, da sie als familieninterne „Hilfslehrerinnen“ nicht qualifiziert genug seien. Pointiert ließe sich das Frauenleitbild der neuen Familienpolitik als das der möglichst hoch qualifizierten, erwerbstätigen Frau mit idealerweise mindestens zwei Kindern beschreiben. Die „gute Mutter“ bleibt nun also nach politischem Willen auch in Westdeutschland, wie früher bereits in der DDR, nicht mehr ausschließlich oder vor allem zu Hause bei ihrem Kind bzw. ihren Kindern, sondern leistet durch Gebärfreudigkeit und möglichst kurz unterbrochene Erwerbstätigkeit, unterstützt vom für maximal zwölf Monate gezahlten Elterngeld, ihren Beitrag zu Bevölkerungs- und Wirtschaftswachstum sowie zur sozialen Sicherung.

Das im konservativen westdeutschen Wohlfahrtsstaatsregime über lange Zeit hinweg in der Familienpolitik erfolgte Festhalten am männlichen Ernährermodell lässt somit stark nach, denn das damit verbundene, traditionelle Frauen- und Familienleitbild passt nicht recht zur politisch angestrebten und ökonomisch notwendigen Steigerung der Frauen- bzw. Müttererwerbstätigkeit. Auf der politischen Agenda steht damit hinsichtlich der Erwerbsbeteiligung der Geschlechter eine Orientierung am Zwei-Verdiener-Modell (vgl. Ostner 2006), das, so die Interpretation Mary Dalys (2004: 143), „geschlechtsneutral“ ist: es 
problematisiere nicht mehr die geschlechtliche Ungleichheit, sondern konstruiere Frauen und Männer als voneinander ökonomisch unabhängige Arbeitskräfte. Ausgeblendet wird dabei jedoch die historisch konstituierte geschlechtliche Arbeitsteilung in privaten und öffentlichen Räumen, die im Zwei-Verdiener-Modell keineswegs per se aufgehoben ist. Inwiefern dem bisherigen familienpolitischen Modell der Ernährerorientierung zugehörige Maßnahmen wie das Ehegattensplitting, das ja vor allem familien- und haushaltsorientierte Frauen im Blick hat, in der realisierten „nachhaltigen Familienpolitik" noch weiter Bestand haben werden, bleibt abzuwarten. Festzuhalten ist jedenfalls bereits jetzt, dass die autonome Frauen- und Gleichstellungspolitik, die auf die Ermöglichung einer Vielfalt von weiblichen Lebensentwürfen mit und ohne Kind(er) und auf Gleichheit und Gerechtigkeit zwischen den Geschlechtern zielt, im Zuge des Erstarkens pronatalistischer Orientierungen und „a move towards gender neutrality“ (Daly 2004: 143) in der Politik rückgebaut wird.

Im Fokus der Expertisen zur „nachhaltigen Familienpolitik“ ebenso wie der neuen familienpolitischen Maßnahmen stehen wesentlich die Frauen als (potenzielle) Mütter, während Männer als (potenzielle) Väter weit weniger politische Aufmerksamkeit erregen. So weisen Bertram u.a. lediglich darauf hin, dass die von ihnen referierten unterschiedlichen Motivlagen zur Vereinbarkeit von Beruf und Familie nicht nur für Frauen gälten, sondern auch für Männer, im Hinblick auf diese „im Regelfall jedoch nicht untersucht“ würden (Bertram u.a. 2005: 14). Ähnlich knapp werden Männer im Gutachten von Rürup/ Gruescu thematisiert, indem sie im Fazit darauf hinweisen, dass die „Ausschöpfung des 'Betreuungspersonals' von Männern"s die Erwerbstätigkeit von Frauen erhöhen könnte. Ohne aber näher auf die dafür notwendigen institutionellen und kulturellen Veränderungen einzugehen, konstatieren sie überzeugt: „Eine nachhaltige Familienpolitik ist also Kinder-, Frauen- und Männerpolitik.“ (Rürup/Gruescu 2003: 75)

Faktisch handelt es sich bei der „nachhaltigen Familienpolitik“ jedoch, auch entgegen anders lautender politischer (Gleichstellungs-)Rhetorik der rot-grünen wie rot-schwarzen Bundesregierung, um eine kindorientierte Politik, die sich vor allem an Frauen als (potenzielle) Mütter richtet. Die Frauen- und Gleichstellungspolitik hat seit der Neuentdeckung der Familienpolitik zu Zeiten der rot-grünen Bundesregierung hingegen kaum noch einen eigenständigen Stellenwert im politischen Handeln bzw. scheint nur dann noch zu interessieren, wenn sie der Erfüllung pronatalistischer Ziele dient (vgl. Kahlert 2006) - und eine explizite Männerpolitik, die der Gleichstellung der Geschlechter in Beruf und Familie dient, ist auch in Zeiten von "Gender Mainstreaming“ noch nicht formuliert. Insofern bleibt das Familien- und Geschlechterleitbild der

5 Gemeint ist wohl das männliche „Betreuungspotenzial“. 
Bundesregierung und der sie beratenden Sozialwissenschaftler doch wesentlich der traditionellen Zuständigkeit von Frauen für die Familie verhaftet. Dennoch tragen die unter demographischem und ökonomischem Druck politisch geförderte Vereinbarkeit von Beruf und Familie und der damit verbundene Paradigmenwechsel vom männlichen Ernährermodell zum Zwei-VerdienerModell sukzessive zur Verwirklichung der Frauenemanzipation bzw. eines egalitären Geschlechterverhältnisses bei. Die dadurch entstehende größere Gleichheit im Geschlechterverhältnis betrifft jedoch zunächst das öffentliche Geschlechterverhältnis; ein Wandel der geschlechtlichen Arbeitsteilung im Privaten, einer wesentlichen Ursache geschlechtlicher Ungleichheit, ist nicht intendiert, könnte jedoch auch eine längerfristige Nebenfolge der pronatalistisch ausgerichteten Politikanstrengungen sein.

\section{7. „Nachhaltige Familienpolitik": Die vertane Chance zur Modernisierung des konservativen deutschen Wohlfahrtsstaats}

Der demographische Wandel könnte durchaus eine Chance für positive Umgestaltungen der europäischen Wohlfahrtsstaaten bieten. Solch positive Umgestaltungen könnten beispielsweise darin bestehen, vorhandene Ungleichheiten in sozialer, geschlechtlicher und ethnischer Hinsicht abzubauen, also erneut an ursprüngliche wohlfahrtsstaatliche Ziele anzuknüpfen und „mehr Gleichheit“" zu wagen. Die vorherigen Ausführungen haben allerdings gezeigt, dass die in Deutschland neu eingeführte pronatalistische Politik dieses Ziel nicht auf der Agenda hat, sondern vielmehr bestehende Ungleichheiten reproduziert und konserviert.

Wäre etwa die Verwirklichung von Gleichheit in den privaten Geschlechterverhältnissen explizit politisch gewollt, so böte sich in familienpolitischer Hinsicht das „proegalitäre Modell“ (Gauthier 1996: 204) an, das an der Veränderung der Geschlechterrollen und der geschlechtlichen Arbeitsteilung auch im Privaten ansetzt - und sich zudem auch positiv auf die Entwicklung der Geburtenrate auszuwirken scheint. Steffen Kröhnert u.a. (2004: 7) stellen nämlich in ihrer international vergleichenden Analyse fest: „Wo Frauen und Männer gleichberechtigt sind, gibt es mehr Kinder." Dabei verstehen sie unter Gleichberechtigung die Partizipation am gesellschaftlichen Leben und machen dies an höheren Bildungsabschlüssen und der Erwerbsbeteiligung von Frauen fest. Auch wenn dieses Gleichheitsverständnis etwas anders als das von Gauthier ausgerichtet ist, so zeigt die Analyse von Kröhnert u.a. aber, dass proegalitäre Familienpolitik sowohl ein „traditionelles“ Ziel des Wohlfahrtsstaats - mehr Gleichheit zwischen den Geschlechtern - einlösen als auch zur Steigerung bzw. Stabilisierung der Geburtenrate auf vergleichsweise hohem Niveau beitragen kann. In gleichheitsbezogener Perspektive wird mit der pronatalistischen Politik in Deutschland also eine „Chance für positive Umgestal- 
tungen“ vertan - wohl nicht nur in Bezug auf die geschlechtliche Ungleichheit.

Die deutsche Antwort auf die demographische Frage ist letztlich auch in sozialer und ethnischer Hinsicht eine strukturkonservative, ganz im Einklang mit dem bisherigen Wohlfahrtsstaatsregime stehende: Demographisch mobilisiert werden sollen im gesellschaftlichen Schrumpfungsprozess aus ökonomischen Gründen insbesondere hoch qualifizierte Frauen und Männer. Diese Menschen mit hohem sozialem Status haben in Deutschland bisher zumeist keinen Migrationshintergrund, im Gegenteil: die Ergebnisse der PISA-Studien zeigen, dass Migrationserfahrungen in Deutschland häufig mit niedrigem sozialem Status einhergehen und eine zentrale Ursache von Bildungsungleichheit darstellen. Die nationale Herkunft scheint in Deutschland jedoch grundsätzlich ein „Qualitätsmerkmal“ der Bevölkerung zu sein - wie beispielsweise die schon ein wenig zurückliegende Diskussion um „Kinder statt Inder“ gezeigt hat: Auch in dieser Debatte wurde letztlich einheimische Geburtenförderung gegenüber der Anwerbung hoch qualifizierter Arbeitskräfte aus dem Ausland priorisiert. Zwar ist die demographische Frage erkennbar eine globale Frage. Ihre politischen Lösungsversuche sind aber nach wie vor nationalgesellschaftlich beschränkt.

\section{Literatur}

Allmendinger, Jutta/Dressel, Kathrin (2005): Familien auf der Suche nach der gewonnenen Zeit, in: Aus Politik und Zeitgeschichte 55 (B23-24), S. 24-29.

Baumert, Jürgen/Schümer, Gundel (2001): Familiäre Lebensverhältnisse, Bildungsbeteiligung und Kompetenzerwerb, in: Deutsches PISA-Konsortium (Hrsg.), PISA 2000. Basiskompetenzen von Schülerinnen und Schülern im internationalen Vergleich, Opladen, S. 323-410.

Becker, Rolf/Lauterbach, Wolfgang (2004): Dauerhafte Bildungsungleichheiten - Ursachen, Mechanismen, Prozesse und Wirkungen, in: Dies. (Hrsg.), Bildung als Privileg? Erklärungen und Befunden zu den Ursachen der Bildungsungleichheit, Wiesbaden, S. 9-40.

Bertram, Hans/Rösler, Wiebke/Ehlert, Nancy (2005): Nachhaltige Familienpolitik. Zukunftssicherung durch einen Dreiklang von Zeitpolitik, finanzieller Transferpolitik und Infrastrukturpolitik. Gutachten im Auftrag des Bundesministeriums für Familie, Senioren, Frauen und Jugend, Berlin.

BMFSFJ (Bundesministerium für Familie, Senioren, Frauen und Jugend) (2005): Familienpolitik der Bundesregierung, in: Das Online-Familienhandbuch (http://www.familienhandbuch.de/ cmain/f_Programme/a_Familienpolitik/s_399.html download: 02.10.2006).

BMFSFJ (Bundesministerium für Familie, Senioren, Frauen und Jugend) (2006): Grundsätze einer nachhaltigen Familienpolitik, Berlin (http://www.bmjsfi.de/Politikbereiche/familie,did=20172.html download: 24.09.2006).

BMFSFJ u.a. (Bundesministerium für Familie, Senioren, Frauen und Jugend / Institut der deutschen Wirtschaft Köln (IW) / Bundesverband der Deutschen Industrie e.V.) (Hrsg.) (2004): Bevölkerungsorientierte Familienpolitik - ein Wachstumsfaktor, Bonn.

BMGS (Bundesministerium für Gesundheit und Soziale Sicherung) (Hrsg.) (2003): Nachhaltigkeit in der Finanzierung der Sozialen Sicherungssysteme. Bericht der Kommission, Berlin.

Bundesregierung (2006): Stellungnahme zum Siebten Familienbericht „Familie zwischen Flexibilität und Verlässlichkeit. Perspektiven für eine lebenslaufbezogene Familienpolitik“, (http://www.bmfsfj.de/Politikbereiche/familie,did=75160.html download 24.09.2006). 
Daly, Mary (2004): Changing Conceptions of Family and Gender Relations in European Welfare States and the Third Way. In: Lewis, Jane/Surender, Rebecca (eds.): Welfare State Change. Towards a Third Way?, Oxford, S. 135-154.

Deutscher Bundestag (2002): Schlussbericht der Enquete-Kommission „Demographischer Wandel - Herausforderungen unserer älter werdenden Gesellschaft an den Einzelnen und die Politik", BT-Drucksache 14/8800, Berlin.

Deutsches PISA-Konsortium (Hrsg.) (2001): PISA 2000. Basiskompetenzen von Schülerinnen und Schülern im internationalen Vergleich, Opladen.

Esping-Andersen, Gøsta (1996): Welfare States without Work: the Impasse of Labour Shedding and Familialism in Continental European Social Policy. In: Esping-Andersen, Gøsta (ed.): Welfare States in Transition. National Adaptations in Global Economies, London, S. 66-87.

Gauthier, Anne Hélène (1996): The State and the Family. A Comparative Analysis of Family Policies in Individualized Countries, Oxford.

Geißler, Rainer (2005): Die Metamorphose der Arbeitertochter zum Migrantensohn. Zum Wandel der Chancenstruktur im Bildungssystem nach Schicht, Geschlecht, Ethnie und deren Verknüpfungen. In: Berger, Peter A./Kahlert, Heike (Hrsg.): Institutionalisierte Ungleichheiten. Wie das Bildungswesen Chancen blockiert, Weinheim/München, S. 71-100.

Hakim, Catherine (2000): Work-lifestyle Choices in the 21st Century: Preference Theory, Oxford.

Kahlert, Heike (2006): Emanzipation in der schrumpfenden Gesellschaft. Der Geburtenrückgang im Fokus der aktuellen Debatten über den demographischen Wandel im deutschen Wohlfahrtsstaat. In: Berliner Debatte Initial 17 (3), S. 9-23.

Kaufmann, Franz-Xaver (2005): Schrumpfende Gesellschaft. Vom Bevölkerungsrückgang und seinen Folgen, Frankfurt/Main.

Kommission (Kommission der Europäischen Gemeinschaften) (2005): Grünbuch „Angesichts des demografischen Wandels - eine neue Solidarität zwischen den Generationen “. Brüssel, KOM (2005) 94 endgültig (http://europa.eu.int/comm/employment_social/social_situation/ green_paper_de.html download 08.09.2006)].

Kröhnert, Steffen/van Olst, Nienke/Klingholz, Reiner (2004): Emanzipation statt Kindergeld. Wie sich die unterschiedlichen Kinderzahlen in den Ländern Europas erklären, (http://www.berlin-institut.org/pdfs/emanzipation_oder_kindergeld.pdf download 05.01.2006).

Oestreich, Heide (2005): Surfen auf dem Megatrend, in: die tageszeitung vom 15.09.2005, S. 11.

Ostner, Ilona (2006): Paradigmenwechsel in der (west)deutschen Familienpolitik. In: Berger, Peter A./Kahlert, Heike (Hg.): Der demographische Wandel. Chancen für die Neuordnung der Geschlechterverhältnisse. Frankfurt, New York, S. 165-199.

Rürup, Bert/Gruescu, Sandra (2003): Nachhaltige Familienpolitik im Interesse einer aktiven Bevölkerungsentwicklung. Gutachten im Auftrag des Bundesministeriums für Familie, Senioren, Frauen und Jugend, Berlin.

Sachverständigenkommission (Sachverständigenkommission Siebter Familienbericht) (2005): Familie zwischen Flexibilität und Verlässlichkeit. Perspektiven für eine lebenslaufbezogene Familienpolitik. Siebter Familienbericht im Auftrag des Bundesministeriums für Familie, Senioren, Frauen und Jugend, Berlin.

Schmidt, Renate (2005): Vorwort, in: Bertram, Hans/Rösler, Wiebke/Ehlert, Nancy: Nachhaltige Familienpolitik. Zukunftssicherung durch einen Dreiklang von Zeitpolitik, finanzieller Transferpolitik und Infrastrukturpolitik. Gutachten im Auftrag des Bundesministeriums für Familie, Senioren, Frauen und Jugend, Berlin, S. 3.

Schmidt, Renate/Sagenschneider, Marie (2004): Familienministerin warnt vor „Bildungskatastrophe“. Interview mit Renate Schmidt, Bundesfamilienministerin (SPD), DeutschlandRadio Berlin vom 21.04.2004 (http://www.dradio.de/dlr/sendungen/interview_dlr/257448/ download 27.05.2006).

Siems, Dorothea (2004): Für mehr Kinder bricht Renate Schmidt Tabus, in: Die Welt vom 15.09.2004 (http://www.welt.de/data/2004/09/15/332438.html?prx=1 download 27.05.2006).

Wissenschaftlicher Beirat (Wissenschaftlicher Beirat für Familienfragen) (2002): Die bildungspolitische Bedeutung der Familie - Folgerungen aus der PISA-Studie, Stuttgart. 